\title{
Modeling and Visualization of Flower Color Patterns
}

\author{
Ning Zhou \\ Department of Computer Science and Technology \\ Tsinghua University, China \\ zhoun03@mails.tsinghua.edu.cn \\ Jiaxin Wang \\ Department of Computer Science and Technology \\ Tsinghua University, China \\ wjx@mail.tsinghua.edu.cn
}

\author{
Weiming Dong \\ Project ALICE \\ INRIA Lorraine, France \\ Weiming.Dong@loria.fr \\ Jean-Claude Paul \\ INRIA, France / Tsinghua University, China \\ paul@mail.tsinghua.edu.cn
}

\begin{abstract}
Flowers are familiar in virtual scenes, however, the design of flower patterns is still mainly done by hand. To produce a number of flower color patterns required by a large scene can be very labor-consuming. In this paper, we present a biologically-motivated algorithm for modeling and visualization of flower color patterns. It is able to produce various flower color patterns with little user interaction. In our system, pigmentation is simulated by a modified reaction-diffusion system, and the simulation function is controlled by a few experiential parameters. Thus it can be adjusted to produce various flower color patterns widely observed in the real world. Furthermore, our algorithm can be easily embedded into other advanced shading models to improve the quality of their rendering results. We present an image-based texture generation method as an example.
\end{abstract}

\section{Introduction}

Flower color patterns, exhibiting various pigmentation and tissue structure influence, are among the most beautiful figures in the natural world. Simulating these patterns has been proved to be an interesting and important challenge for computer graphics. When light interacts with flower petals, several optical effects occur, namely reflection, transmission and absorption. The color we observe on the flowers is formed by the reflective light from the petals, and moreover, the reflection is determined by the absorption, which is real determinant for the appearances of the flowers [5]. Pigments in the flower petals absorb light of certain wavelengths. One pigment has a specific light absorption spectrum, which is mainly determined by its molecule structure. Both the absorbance and distribution of pigments generally decide the flower color pattern.

In this paper, we introduce an efficient system for modeling and visualization of flower color patterns. Our goal is to build a connection between the user-controllable image synthesis and real botanical patterns, that is to provide a flexible control allowing users to model a wide variety of biologically plausible flower color patterns. With our system, users can generate realistic flower patterns from corresponding pigment data without the requirement of professional painting skills or any special data acquisition device. Note that we pay more attention to simplifying the modeling process of the flower color pattern and enhancing its diversity than improving the rendering techniques.

The workflow of our system can be divided into three steps. First, we analyze the components of the flower color, and decompose it into two parts: pattern information and environment affection. Then we calculate the pattern information based on the pigment distribution with the reactiondiffusion system, which has been used in natural pattern formation for a long time [13]. The pigment manipulation and transportation are also dependent on the vascular structure. Thus, we modified the standard reaction-diffusion system to fit this demand. The third step is to integrate pattern information into the predefined environment. Combining with rendering systems, our results also can be used as data for texture functions or directly as texture images to render realistic scenes. The whole process from user input to further application, such as rendering, is shown in Figure 1.

The remainder of the paper is organized as follows. Section 2 reviews related work and gives some background botanical knowledge. Section 3 explains how to generate the pigment distribution map with the reaction-diffusion equations. Section 4 discusses the visualization of the simulated flower color pattern. Section 5 analyzes the results, and section 6 draws the conclusion. 


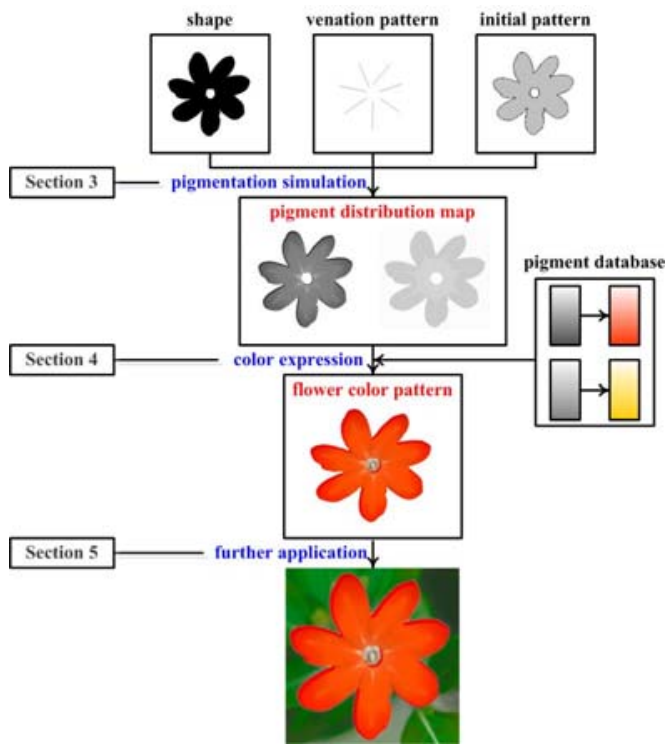

Figure 1. Illustration of our system framework

\section{Related Work}

Simulation of patterns on living organisms has been studied by researchers of biology, mathematics and computer graphics for a long time. Color patterns are broadly observed in appearance of natural objects, such as plants, animals and insects. It is necessary to simulate the color patterns in order to generate realistic images of those objects. In previous work, attention has been paid to mammalian, fish, sea shell, tree bark, etc. However, pattern formation of flowers is still blank.

Instead of making strict biologically-based simulations, mathematical models have been used to simulate the color pattern formation in nature. Although there is no final conclusion on their validity, they are able to produce visual plausible results with relative higher performance. Previous work includes reaction-diffusion system used in animal fur simulation [18], sea shell pigmentation [4], and fish skin pattern formation [14]; the Clonal Mosaic model for mammalian coat pattern formation [19]; a semi-empirical model for bark generation [8]; an open diffusion limited aggregation model for lichen growth and propagation simulation [3]; and a biologically-motivated algorithm for generating venation patterns [15].

For synthesizing realistic images, various botanicallybased modeling and rendering systems have been developed to support the design and visualization of plants. Lintermann and Deussen [9] present a modeling method for generating branching objects. Mündermann et al. [12] extended the concept of branching structure and developed an interactive method for modeling lobed leaves. Ijiri et al. [7] supplied a convenient tool motivated by biology knowledge for flower geometry modeling, which can preserve correct botanical structures. Govaerts et al. [6] developed a leaf model using geometrical primitives to represent the different types of cells found in the foliar tissues. Baranoski and Rokne [1] presented an algorithmic reflectance and transmittance model for plant tissue oriented to computer graphics applications. Wang et al. [20] developed a framework for rendering of plant leaves in real-time with global illumination. The spatially-variant BRDFs and BTDFs employed to describe leaf appearance are stored in a set of parameter maps. This method is efficient and produces very realistic results. However, it is tightly limited by measured data and will be inconvenient when plants with many patterns are required in one scene.

\section{Pigmentation Simulation}

In this section, we explain how to simulate the pigment distribution of flower petals. We first input the information of petal shape, venation, and initial distribution. Then we simulate the pigmentation with a reaction-diffusion system, and save the normalized simulation result in a greyscale map for the next step.

There are two kinds of flowers: monochrome flowers, and flowers bearing variegation [21]. The latter one often produces near-regular patterns, which we are interested in. Common variegation patterns, including center spot, marginal band, ring and radial stripe, are shown in Figure 2. These patterns may appear simultaneously in one flower. The space-time distribution of flower color pigment is the most important determinative factor for flower patterns [21]. To achieve our goal, it is essential to simulate this distribution.
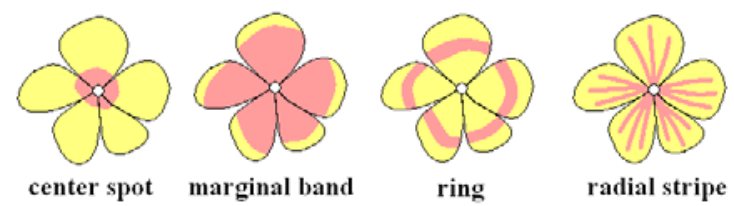

(a) Sketch map of common flower color patterns in nature

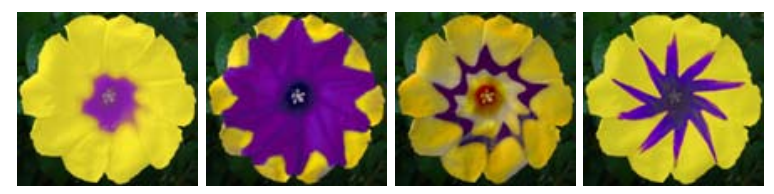

(b) Corresponding result samples

Figure 2. Flower color patterns. 


\subsection{Preliminaries}

The input for our system consists of: (1) the initial state (shape and venation information of the flower, and the initial pigment distribution), (2) parameters characterizing the spread and interplay of pigments, and (3) functions and parameters defining flower color expression.

The shape is specified by the user as a black-white image that defines the petal's range. The venation pattern is given as a set of vein nodes, including a vein root and vein width. According to the canalization hypothesis [2], the vein is composed of cells with higher capability in auxin transportation. We assume that the connectivity coefficient between two adjacent tissue cells equals to $p_{m m}$; the connectivity coefficient between a vascular cell and an adjacent tissue cell is $p_{m v}$; the connectivity coefficient between two adjacent vascular cells is $p_{v v}$. In our system, we set $p_{m m}=1, p_{m v}=2$ and $p_{v v}=10$. Moreover, we define that the connectivity coefficient between two non-adjacent cells is directly proportional to the vein width and is inversely proportional to the distance between them. Then, we define the transportation function between two cells $a$ and $b$ as follows:

$$
I(a, b)=\left\{\begin{array}{l}
p_{m m} /\|a, b\| \\
p_{m v} \cdot w /\|a, b\| \\
p_{v v} \cdot \bar{w}_{a b} /\|a, b\|
\end{array}\right.
$$

In (1a), neither $a$ or $b$ is a vascular cell; in (1b), either $a$ or $b$ is a vascular cell and its vein width is $w$; in (1c), $a$ and $b$ are both vascular cells and connected by the vein with average width $\bar{w}_{a b}$.

The leaf shape and venation pattern can be drawn or extracted from photos. Artificial methods such as [15] can also be used to generate a vascular system. Normalized pigment distribution can be represented by a greyscale map called pigment distribution map. Each kind of pigment corresponds to one map. The initial pigment distribution is given by users.

\subsection{Pigment Composition Mechanism}

We use reaction-diffusion equations to describe the interaction among different pigments. Based on the assumption that the pigment composition of one cell can be calculated from its neighbors, we can simulate the pigmentation of following moments in the growing process if an initial state and transportation kinetics are given. The initial state can be freely assigned by the user. For convenience, we use an uniform distribution map as the initial state in our experiments.

In the two-pigment case, we assume $A_{t}$ and $B_{t}$ are the time-dependent concentrations of two pigments $A$ and $B$.
The reaction-diffusion equations describing the general relation between $A_{t}$ and $B_{t}$ are given by

$$
\left\{\begin{array}{c}
\frac{\partial A_{t}}{\partial t}=F\left(A_{t}, B_{t}\right)+D_{A} \nabla^{2} A_{t} \\
\frac{\partial B_{t}}{\partial t}=G\left(A_{t}, B_{t}\right)+D_{B} \nabla^{2} B_{t}
\end{array}\right.
$$

Where $F$ and $G$ are the kinetics, and $D_{A}, D_{B}$ is the positive constant diffusion coefficients. Different kinetics have been invented to describe the pattern formation process in nature. In our system, we employ the following kinetics designed by Schnakenberg [13]:

$$
\left\{\begin{array}{l}
F\left(A_{t}, B_{t}\right)=k_{1}-k_{2} A_{t}+k_{3} A_{t}^{2} B_{t} \\
G\left(A_{t}, B_{t}\right)=k_{4}-k_{3} A_{t}^{2} B_{t}
\end{array}\right.
$$

$k_{1}, k_{2}, k_{3}$ and $k_{4}$ are positive empirical constants [11].

The Laplacian is $\nabla^{2} A=\frac{\partial^{2}}{\partial x^{2}} A+\frac{\partial^{2}}{\partial y^{2}} A$. It is difficult to compute the Laplacian in the continuous domain, thus we discretize the continuous plane to a grid, and then we can use the finite difference to approximate the Laplacian:

$$
l_{i, j}=\frac{1}{4}\left(u_{i+1, j}+u_{i-1, j}+u_{i, j+1}+u_{i, j-1}\right)-u_{i, j}
$$

where $l_{i, j}$ is the Laplacian at point $(i, j) . u_{i, j}$ is the value at point $(i, j)$.

To represent the effect of vein, we modify the neighbors of $(i, j)$ in (4) with auxin transportation function (1): $u_{m, n}^{(i, j)}=v I((i, j),(m, n)) \cdot u_{m, n}$, where $v$ is the proportion coefficient of venation influence. Then

$$
l_{i, j}^{(u)}=\frac{1}{4}\left(u_{i+1, j}^{(i, j)}+u_{i-1, j}^{(i, j)}+u_{i, j+1}^{(i, j)}+u_{i, j-1}^{(i, j)}-4 u_{i, j}\right)
$$

So substitute $\left(4^{\prime}\right)$ to $(2)$, the modified discrete form reaction-diffusion equations on a grid can be rewritten as:

$$
\left\{\begin{array}{l}
\Delta A_{i, j}=S_{A}\left(a_{i, j}^{2} b_{i, j}-K_{A} a_{i, j}+\beta\right)+D_{A} l_{i, j}^{(a)}(5 \mathrm{a}) \\
\Delta B_{i, j}=S_{B}\left(1-K_{B} a_{i, j}^{2} b_{i, j}\right)+D_{B} l_{i, j}^{(b)}
\end{array}\right.
$$

where

$\Delta A_{i, j}$ : concentration change of pigment $A$ at $(i, j)$;

$a_{i, j}$ : concentration of pigment $A$ at $(i, j)$;

$l_{i, j}^{(a)}$ : influence to the concentration of pigment $A$ from neighbors of $(i, j)$;

$S, K, D, \beta$ : constants depending on transportation kinetics. If we use the kinetics mentioned in (3), the constants in (5) will be $S_{A}=k_{3}, K_{A}=\frac{k_{2}}{k_{3}}, \beta=\frac{k_{1}}{k_{3}}$, and $S_{B}=k_{4}$, $K_{B}=\frac{k_{3}}{k_{4}}$.

The reaction-diffusion system is able to simulate both the decrease of inherent pigment and the increase of subsequent pigment during the growth. The parameter adjustment technique can be found in [18]. Our modification embeds the effect of vascular transportation into the primary mechanics, 
Table 1. Symbol and meaning of the parameters in our system

\begin{tabular}{c|l}
\hline Symbol & Meaning \\
\hline$S$ & proportion coefficient between \\
& diffusion and reaction \\
$D$ & reaction coefficient \\
$k$ & diffusion coefficient \\
$\beta$ & dissipation coefficient \\
& responsible for slight irregularity \\
$v$ & proportion coefficient of venation influence \\
$c$ & normalized concentration of pigments \\
\hline
\end{tabular}

and leads to more control of the final results. To simulate the interplay of more than two pigments, more complex kinetics from [11] should be used. The influence of the auxin transportation function is demonstrated in Figure 3(b).
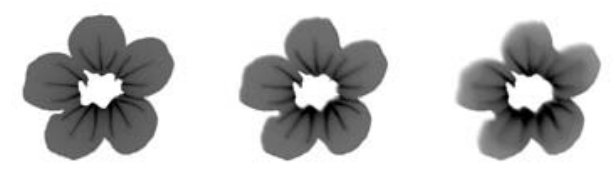

(a)
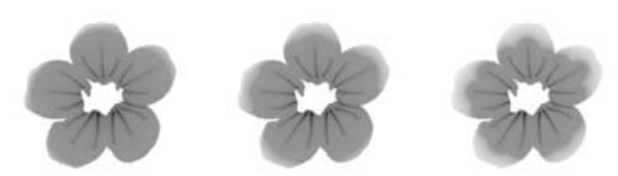

(b)

Figure 3. Different patterns generated by parameter adjustment. Figure $3(a)$ shows the influence of the parameter $D$. From left to right, $D=0.1,0.3,0.5$. Figure $\mathbf{3}(\mathbf{b})$ shows the influence of auxin transportation function. From left to right, $t=1,2,5$

We define a certain concentration value as standard concentration $C_{0}$. The pigment concentration calculated by the modified reaction-diffusion equations are normalized by $c=C / C_{0}$, and then stored in the greyscale pigment distribution map. In our system, we usually use the highest concentration in the pigment color database as standard concentration. Symbols and meanings for all the parameters used for pigmentation simulation are shown in Table 1. After the pigment distribution map is computed, it will be painted with pigment color information.

\section{Flower Color Expression}

To paint the greyscale pigment distribution map generated in the previous step, we first construct a pigment database to store the absorb color data of all pigments of different concentrations, and some other information which is not related with the pigment distribution map, such as the lighting environment. After these pre-computation is finished, we calculate the color pattern of the petal based on the pigment distribution map in runtime.

From the physics point of view, flower color is determined by light absorption of pigments, which is a function of wavelength. The wavelength range of visible light extends approximately from $\lambda_{\min }=380 \mathrm{~nm}$ to $\lambda_{\max }=$ $770 \mathrm{~nm}$. Thus, the tristimulus values $X$ of an energy spectrum $P(\lambda)$ sensed by human eyes are

$$
X^{(k)}=\int_{\lambda_{\min }}^{\lambda_{\max }} R(\lambda) \bar{x}_{k}(\lambda) d \lambda, k=1,2,3,
$$

where $\lambda$ is the wavelength. Here we assume there is no interplay among the color performances of different pigments.

\subsection{Pre-computation of pigment color performance}

The standard pigment color performance is calculated based on measured data under standard conditions. Suppose there is a pigment $p$ with standard absorption spectrum $A(\lambda)$. The luminance spectrum $L(\lambda)$ represents the energy of incident light, which is considered to be a constant in the simulation process. The transmittance spectrum is $T(\lambda)$, which depends on the material of the petal. It varies greatly due to the species, and can be somewhat affected by the pigment concentration. As the influence of pigment concentration to transmittance is not obvious, in our system, we use the measure data from several typical plant tissue types to make an approximation of the transmittance [17][16], and consider it as constant for a certain flower. Then the reflection spectrum $R(\lambda)$ can be calculated by $R(\lambda)=L(\lambda)-T(\lambda)-A(\lambda)$. We divide the right side of the above equation into two parts: $L(\lambda)-T(\lambda)$ and $A(\lambda)$, and define their corresponding tristimulus values as

$$
\begin{gathered}
X_{0}^{(k)}=\int_{\lambda_{\min }}^{\lambda_{\max }}(L(\lambda)-T(\lambda)) \bar{x}_{k}(\lambda) d \lambda \\
X_{A}^{(k)}=\int_{\lambda_{\min }}^{\lambda_{\max }} A(\lambda) \bar{x}_{k}(\lambda) d \lambda
\end{gathered}
$$

Substitute (7a) and (7b) into (6), it can be rewritten to a composition of two parts

$$
X^{(k)}=X_{0}^{(k)}-X_{A}^{(k)}, k=1,2,3 .
$$


The standard chromaticity coordinates (corresponding to standard concentration $C_{0}$ ) of the pigment are:

$$
(x, y)=\left(\frac{X^{(1)}}{X^{(1)}+X^{(2)}+X^{(3)}}, \frac{X^{(2)}}{X^{(1)}+X^{(2)}+X^{(3)}}\right)
$$

For pigment of normalized concentration $c$, according to Beer's law [10], its absorption spectrum approximately follows linear relationship: $A_{c}(\lambda) \approx c \cdot A(\lambda)$, so its tristimulus values are $X_{c}^{(k)}=X_{0}^{(k)}-c \cdot X_{A}^{(k)}=X^{(k)}+(1-c) \cdot X_{A}^{k}$, $k=1,2,3$.

By Equation 6', we pre-compute values which have no relation with pigment concentration based on measure data of lighting condition and transmittance indices. Only the values related with pigment concentration need to be computed at runtime.

\subsection{Runtime flower color expression}

For single-pigment flowers, the flower color expression is determined by the absorption spectrum of that pigment.

Even only with one single pigment, the flower color varies and forms special patterns because of the inhomogeneous distribution. With the same pigment distribution map, if we change the type of pigment, we can get flowers with similar pattern but different colors. Figure 4 shows some single-pigment samples generated with our system based on the same pigment distribution map but with different pigment. Change of concentration has stronger influence on saturation than on hue, and it has a linear relation with lightness. Figure 5 illustrates how the color expression of the same pigment varies because of different concentration.

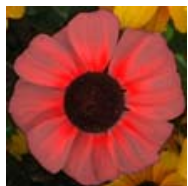

(a)

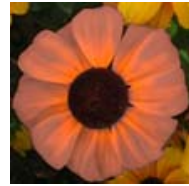

(b)

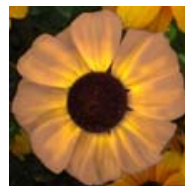

(c)

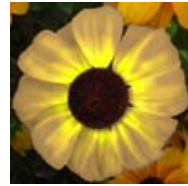

(d)

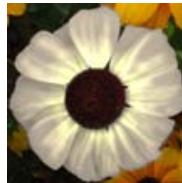

(a)

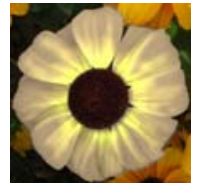

(b)

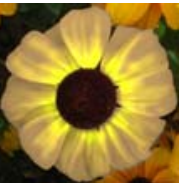

(c)

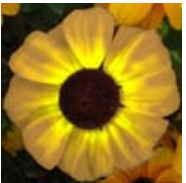

(d)
Figure 5. Effects of concentration. The flower color changes when the standard concentration $C_{0}$ changes. From $5(\mathrm{a})$ to $\mathbf{5}(\mathrm{d}), C_{0}$ is set to $0.25,0.5,0.75$ and 1 .

its tristimulus values are:

$$
X^{\prime(k)}=X_{0}^{(k)}-\sum_{i=1}^{n} c_{i} \cdot X_{A_{i}}^{(k)}, k=1,2,3 .
$$

In case of mixed pigments, the relationship between color and concentration $c_{i}$ is not linear anymore, though each separate pigment keep its linear relationship. Some mixed pigments samples generated with our system are shown in Figure 6. Figure 6(a) and 6(b) are distribution maps of two different pigments existing in the flower. Figure $6(\mathrm{c})$ and $6(\mathrm{~d})$ are results based on same distribution maps but with different pigment contents.

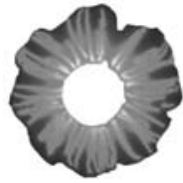

(a)

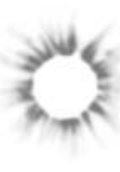

(b)

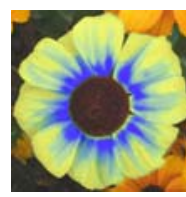

(c)

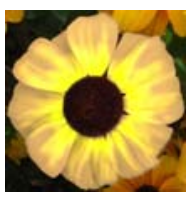

(d)
Figure 6. Mixed pigments color expression. 6(a) and 6(b) are the distribution maps of the two pigments in the flower petal. 6(c) and 6(d) are flower color patterns generated by assigning different kinds of pigments to $6(\mathrm{a})$ and 6(b).

\section{Results}

We implemented our system on a PC with $\mathrm{P} 43.2 \mathrm{GHz}$ CPU, 1GB RAM and a Geforce 7800 GT graphics card. In this section, we report flower color patterns generated with our system, and rendering results using reflectance data acquired with our algorithm. The performance of our system is shown in Table 2.

The primary contribution of our work is a sufficient tool for generating and editing flower color patters. Given a monochrome flower, our algorithm is able to change its hue, pigment. Several pigments cooperate to show a color which is different from all of their original color expressions.

Suppose there are pigments $p_{i}$ of concentration $c_{i}$, and their typical absorption spectra are $A_{i}(\lambda), i=1$ to $n$. Then the total absorption spectrum is $A(\lambda)=\sum_{i=1}^{n} c_{i} \cdot A_{i}(\lambda)$, so 
Table 2. Performance statistics for figures

\begin{tabular}{cccc}
\hline $\begin{array}{c}\text { Figure } \\
\text { Number }\end{array}$ & Plant & $\begin{array}{c}\text { Resolution } \\
\text { (pixels) }\end{array}$ & $\begin{array}{c}\text { Compute Time } \\
\text { (seconds) }\end{array}$ \\
\hline $2(\mathrm{~b})$ & Petunia & $549 \times 528$ & 466 \\
3 & Geranium & $430 \times 387$ & 212 \\
$4,5,6$ & Daisy & $275 \times 276$ & 110 \\
\hline
\end{tabular}

and assign different variegation to it. As a simple demonstration, we use an image-based method to generate texture maps with our results. Our algorithm can also be embedded into other models to achieve higher rendering quality.

\section{Conclusion}

We have presented an algorithm for flower color pattern modeling and visualization. Cooperated with reactiondiffusion system and flower color analysis, our system combines pigment distribution simulation and flower color expression together under a unified framework. Our system is able to form a wide variety of biologically plausible flower color patterns, which are widely observed in nature. The result patterns can be used to produce more realistic virtual scenes, and our system can also be directly embedded into other modeling or rendering systems to improve their results. Besides biologically-related simulation and application, our work demonstrates that even though parameters are not available from measure data, an approximate simulation can be made based on a few empirical parameters. Thus, it reveals the ability of visualizing imaginative flowers, with which data acquisition is impossible.

For future work, we want to extend our algorithm to pattern generation for other objects with similar properties.

\section{Acknowledgements}

The authors would like to thank the anonymous reviewers for their thoughtful comments. Thanks to Koen Beets for the proofreading.

\section{References}

[1] G. V. G. Baranoski and J. G. Rokne. An algorithmic reflectance and transmittance model for plant tissue. Computer Graphics Forum, 16(3):141-150, 1997.

[2] T. Berleth, J. Mattsson, and C. S. Hardtke. Vascular continuity, cell axialisation and auxin. Plant Growth Regulation, 32:173-185, 2000.

[3] B. Desbenoit, E. Galin, and S. Akkouche. Simulating and modeling lichen growth. Computer Graphics Forum, 23(3):341-350, 2004.
[4] D. R. Fowler, H. Meinhardt, and P. Prusinkiewicz. Modeling seashells. In Proceedings of SIGGRAPH 1992, pages 379387, New York, NY, USA, 1992. ACM Press.

[5] T. W. Goodwin. Chemistry and Biochemistry of Plant Pigments. Academic Press, London, UK, 1976.

[6] Y. M. Govaerts, S. Jacquemoud, M. M. Verstraete, and S. L. Ustin. Three-dimensional radiation transfer modeling in a dicotyledon leaf. Applied Optics, 35(33):6585-6598, 1996.

[7] T. Ijiri, S. Owada, M. Okabe, and T. Igarashi. Floral diagrams and inflorescences: interactive flower modeling using botanical structural constraints. ACM Trans. Graph., 24(3):720-726, 2005.

[8] S. Lefebvre and F. Neyret. Synthesizing bark. In Proceedings of the 13th Eurographics workshop on Rendering, pages 105-116, Aire-la-Ville, Switzerland, 2002. Eurographics Association.

[9] B. Lintermann and O. Deussen. Interactive modeling of plants. IEEE Comput. Graph. Appl., 19(1):56-65, 1999.

[10] D. L. MacAdam. Color Measurements Theme and Variations. Springer Verlag, Berlin, Germany, 1981.

[11] H. Meinhardt. Models of Biological Pattern Formation. Academic Press, London, UK, 1982.

[12] L. Mündermann, P. MacMurchy, J. Pivovarov, and P. Prusinkiewicz. Modeling lobed leaves. In Proceedings of Computer Graphics International 2003, pages 60-68, Tokyo, Japan, 09-11 July, 2003.

[13] J. D. Murray. Mathematical Biology, Volume II: Spatial Models and Biomedical Applications, pages 71-81. Springer Verlag, Berlin, Germany, 2003.

[14] K. J. Painter. Models for pigment pattern formation in the skin of fishes. IMA Volumes in Maths. \& App., 121(3):59$82,2000$.

[15] A. Runions, M. Fuhrer, B. Lane, P. Federl, A.-G. RollandLagan, and P. Prusinkiewicz. Modeling and visualization of leaf venation patterns. ACM Trans. Graph., 24(3):702-711, 2005.

[16] D. A. Sims and J. A. Gamon. Relationships between leaf pigment content and spectral reflectance across a wide range of species, leaf structures and developmental stages. Remote Sensing of Environment, 81(2002):337-354, 2002.

[17] P. S. Thenkabail, R. B. Smith, and E. D. Pauw. Hyperspectral vegetation indices and their relationships with agricultural crop characteristics. Remote Sens. Environ., 71:158$182,2000$.

[18] G. Turk. Generating textures on arbitrary surfaces using reaction-diffusion. In Proceedings of SIGGRAPH 1991, pages 289-298, New York, NY, USA, 1991. ACM Press.

[19] M. Walter, A. Fournier, and M. Reimers. Clonal mosaic model for the synthesis of mammalian coat patterns. In Graphics Interface, pages 82-91, 1998.

[20] L. Wang, W. Wang, J. Dorsey, X. Yang, B. Guo, and H.-Y. Shum. Real-time rendering of plant leaves. ACM Trans. Graph., 24(3):712-719, 2005.

[21] C.-L. Zhao, W.-M. Guo, and J.-Y. Chen. Formation and regulation of flower color in higher plants (in chinese). Chinese Bulletin of Botany, 22(1):70-81, 2005. 